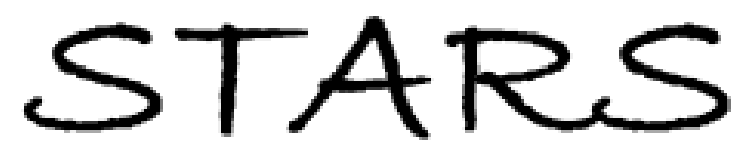

University of Central Florida

STARS

$1-1-2012$

\title{
Cascaded plasmonic metamaterials for phase-controlled enhancement of nonlinear absorption and refraction
}

\author{
Seyfollah Toroghi \\ University of Central Florida \\ Pieter G. Kik \\ University of Central Florida
}

Find similar works at: https://stars.library.ucf.edu/facultybib2010 University of Central Florida Libraries http://library.ucf.edu

This Article is brought to you for free and open access by the Faculty Bibliography at STARS. It has been accepted for inclusion in Faculty Bibliography 2010 s by an authorized administrator of STARS. For more information, please contact STARS@ucf.edu.

\section{Recommended Citation}

Toroghi, Seyfollah and Kik, Pieter G., "Cascaded plasmonic metamaterials for phase-controlled enhancement of nonlinear absorption and refraction" (2012). Faculty Bibliography 2010s. 3394. https://stars.library.ucf.edu/facultybib2010/3394

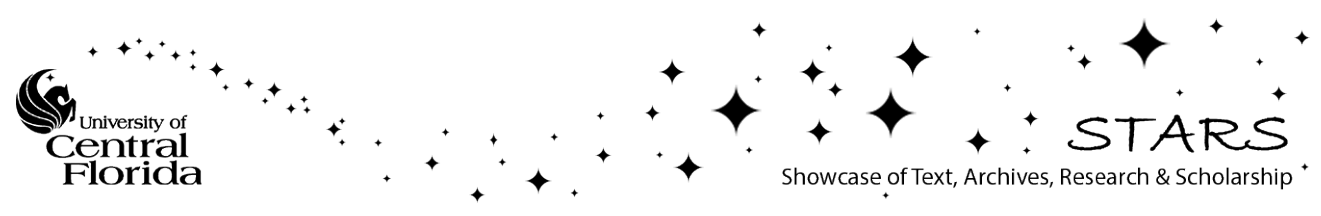




\title{
Cascaded plasmonic metamaterials for phase-controlled enhancement of nonlinear absorption and refraction
}

\author{
Seyfollah Toroghi and Pieter G. Kik \\ CREOL, College of Optics and Photonics, University of Central Florida, 4000 Central Florida Blvd., Orlando, Florida 32816, USA
}

(Received 25 July 2011; revised manuscript received 4 January 2012; published 19 January 2012)

\begin{abstract}
The nonlinear optical properties of plasmon resonant metamaterials consisting of chains of metal nanoparticles are evaluated. Introducing particle size differences along the chains leads to the development of cascaded plasmon resonances exhibiting increased field enhancement and field confinement. The interplay among the different resonances on the structures induces a frequency-dependent enhancement of the nonlinear refractive and absorptive response of the metamaterial, ultimately providing larger nonlinear susceptibility enhancement factors with engineered complex phase. It is shown that cascaded structures can provide a figure of merit for nonlinear absorption that is more than an order of magnitude larger than that obtained in noncascaded structures. The presented approach could lead to new planar and integrated nonlinear optical modulation and switching media with improved performance compared to their noncascaded counterparts.
\end{abstract}

DOI: 10.1103/PhysRevB.85.045432

PACS number(s): 78.67.Sc

\section{INTRODUCTION}

Metallic nanocomposites are of great interest in nanophotonics due to their ability to provide new optical functionality through the use of engineered plasmon resonances and their corresponding enhanced electric fields. One of the most striking effects associated with these enhanced local fields is a dramatic change in the nonlinear optical response of the composite. Harmonic generation, for example, second and third harmonic generation, can be enhanced in such nonlinear metamaterials. ${ }^{1-5}$ In addition, nonlinear optical refraction and absorption are strongly modified in these metamaterials because the enhancement of Kerr-type third-order nonlinearities scales with the fourth power of the electric field enhancement. ${ }^{6}$ While large enhancements in nonlinear susceptibility are readily obtained, achieving improved device performance is nontrivial because the introduction of plasmon resonant elements necessarily contributes to the linear absorption. For this reason, application-specific figures of merit are defined that can take into account any absorption present in the system. This becomes especially important in applications that operate in transmission mode, as is the case in absorptive optical switching. ${ }^{7-9}$ The third-order nonlinear response and corresponding figure of merit (FOM) of several nanostructured metamaterials have been investigated as a function of a variety of structural parameters. ${ }^{10-18}$

A current area of particular interest involves the nonlinear optical response of systems that exhibit coupled plasmon resonances. The interaction of coupled, localized surface plasmons in dimers and particle chains, as well as their relevant spectral properties and field enhancement factors, has been studied extensively. ${ }^{19-29}$ In recent years, a special type of coupled plasmon resonances, known as cascaded plasmon resonances, has received significant attention due to its ability to provide high field enhancement. The use of cascaded resonances could potentially lead to nonlinear metamaterials that exhibit a stronger nonlinear optical response than can be obtained using isolated plasmonic resonators. $\mathrm{Li}$ et al. theoretically showed that cascaded field enhancement occurs in a nanolens configuration consisting of coupled plasmon resonant nanospheres with varying sizes. ${ }^{30}$ Kravets et al. used a structure consisting of three coaxial gold disks on top of one another to show cascaded field enhancement experimentally. ${ }^{31}$ Similar methods may be used to obtain large nonlinear susceptibility, but it is not yet known whether the stronger localized field and the modified frequency response can produce an improved FOM for nonlinear absorption or refraction.

In the present study, we investigate the nonlinear absorptive and refractive response of metamaterials composed of nearfield-coupled spherical silver nanoparticles with a binary size distribution. An example of the resulting cascaded plasmon resonant nonlinear metamaterial is shown in Fig. 1. By varying the size difference between adjacent particles, the plasmon resonant metamaterial is gradually adjusted from the noncascaded to the cascaded regime. This approach enables a systematic investigation of the relative importance of the cascading effect. We show that the cascading leads to an overall enhancement of the FOM for third-order nonlinear absorption and refraction of the metal particles. Complex enhancement factors in arrays containing a single particle size are predominantly imaginary or negative but not positive. In cascaded systems, however, a large positive enhancement factor of the nonlinear optical response is found. This type of phase-optimized design of the nonlinear optical response enhancement in cascaded plasmon resonant metamaterials could lead to the development of new optical switching materials with performance that dramatically exceeds that of their noncascaded counterparts.

\section{METHODS}

We consider two-dimensional arrays of silver nanoparticles of alternating sizes, resulting in a set of nanoparticle chains with relatively small interparticle spacing within each chain and relatively large spacing between chains, as indicated schematically in Fig. 1. To remain well within the quasielectrostatic limit, small particle sizes (diameter $<2.5 \mathrm{~nm}$ ) have been assumed. However, as discussed in more detail later, the approach is not limited to such small sizes and can be scaled up by at least an order of magnitude with similar results. 


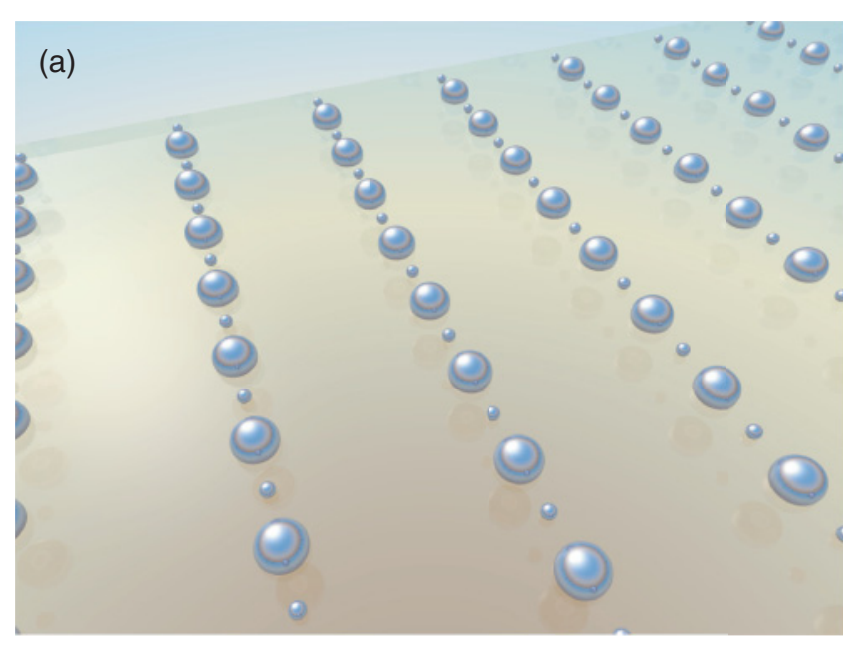

(b)

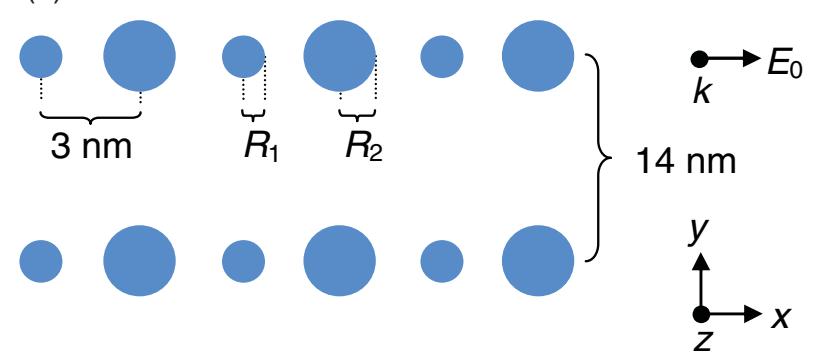

FIG. 1. (Color online) (a) Three-dimensional rendering of a typical cascaded plasmon resonant nonlinear metamaterial, containing regularly spaced silver particles with a binary particle size distribution. (b) Cross-section of one of the simulated cascaded plasmon resonant nonlinear metamaterials, indicating the relevant size and spacing parameters. The structure is excited using an $x$-polarized plane wave propagating in the $z$-direction.

The center-to-center spacing between adjacent particles is held constant at $3 \mathrm{~nm}$, while the interchain spacing is set to $14 \mathrm{~nm}$. The nanoparticles are assumed to be embedded in a host with refractive index of 1.5, representative of, e.g., glass or common polymeric optical materials. The linear optical properties of the silver particles are modeled by a Drude fit to literature data given by $\varepsilon_{\mathrm{Ag}}=\varepsilon_{\infty}-\omega_{p}{ }^{2} /\left(\omega^{2}+i \omega \Gamma\right)$ where $\varepsilon_{\infty}=5.451, \omega_{p}$ $=1.474 \times 10^{16} \mathrm{rad} / \mathrm{s}$, and $\Gamma=8.354 \times 10^{13} \mathrm{~s}^{-1}$. Similar to the assumptions made in earlier publications on cascading by Li et al. ${ }^{30,32}$ this dielectric function does not include the effect of surface scattering that occurs at small particle diameters. The implications of this choice are discussed in greater detail in the results section. Five geometries with different size dissimilarities between adjacent particles are considered, with particle radii $\left[R_{1}(\mathrm{~nm}), R_{2}(\mathrm{~nm})\right]$ of $[1,1],[0.85,1.12],[0.7$, $1.18],[0.55,1.22]$, and $[0.4,1.25]$, corresponding to particle volume ratios $\left(R_{2} / R_{1}\right)^{3}$ of $1,2.3,4.8,11$, and 30 . At the chosen sample thickness of $10 \mathrm{~nm}$, these choices lead a fixed metal fill fraction of $1 \%$, facilitating direct comparison of the results.

Three-dimensional, full-vectorial electric field distributions were simulated using frequency domain finite integration technique simulations. ${ }^{33}$ The structures are illuminated at normal incidence (along the $z$-axis) using plane wave excitation polarized along the nanoparticle chain direction ( $x$-axis). The obtained frequency-dependent electric field distributions are subsequently evaluated numerically to yield effective medium properties. $^{18}$

\section{RESULTS AND DISCUSSION}

Figure 2 shows the calculated linear absorption spectra of the five metamaterials, obtained through the numerical integration of simulated frequency-dependent field distributions using the method described in Ref. 18. For nanoparticle arrays with a single particle size (curve E), two absorption maxima are observed. The main resonance at $432 \mathrm{~nm}$ is due to the resonant excitation of a predominantly dipolar plasmon resonance in the individual nanoparticles. This resonance occurs red-shifted relative to the predicted resonance wavelength of $404 \mathrm{~nm}$ for similarly sized, isolated silver nanoparticles in the same host material as a result of near-field coupling between adjacent nanoparticles. ${ }^{34}$ The second, relatively weak absorption feature at a wavelength of $375 \mathrm{~nm}$ is due to the excitation of a multipolar plasmon resonance on the nanoparticles, as indicated schematically in the inset in Fig. 2. While this mode is not excited significantly in isolated nanoparticles of this size, the inhomogeneous near-field contributions originating from neighboring particles facilitate the excitation of higher-order modes. The introduction of a finite size difference between adjacent particles leads to two main changes in the absorption spectrum. First, the main absorption peak gradually blue-shifts due to modified interparticle coupling. This effect can be understood by considering the limiting case in which the volume of the smaller particle is reduced to zero at a fixed metal fill fraction, resulting in an array of slightly larger nanoparticles at an increased interparticle spacing. This leads to weaker nearest-neighbor coupling and a corresponding reduced redshift. In addition, the introduction of size differences along the array modifies the array periodicity, leading to the appearance of an additional absorption resonance peak at $\sim 395 \mathrm{~nm}$. This

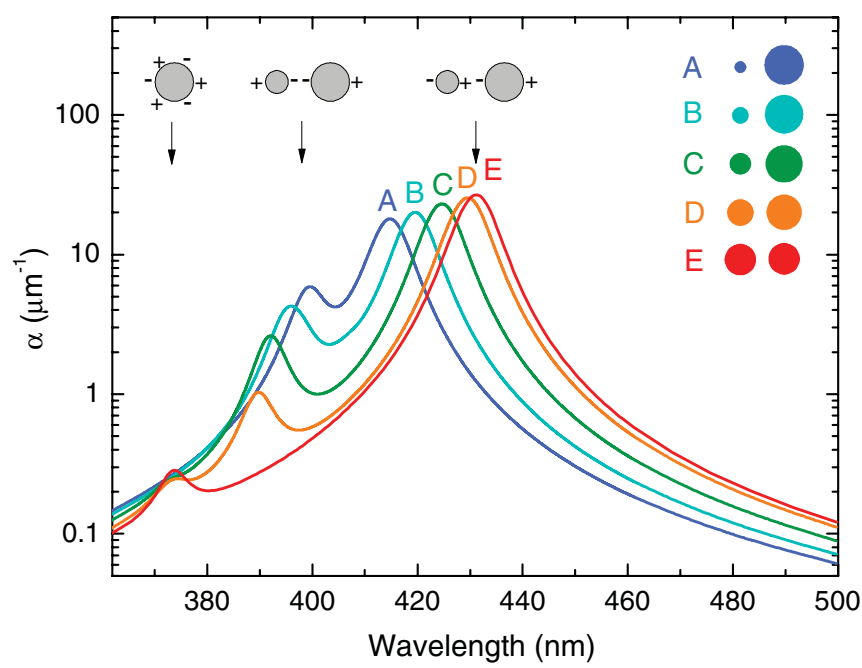

FIG. 2. (Color online) Absorption coefficient for five cascaded plasmon resonant metamaterials with nearest-neighbor volume ratios of $1,2.3,4.8,11$, and 30. A snapshot of the surface charge distribution corresponding to the three main resonance features is indicated schematically. The legend shows the corresponding unit cell of the periodic metamaterial. 

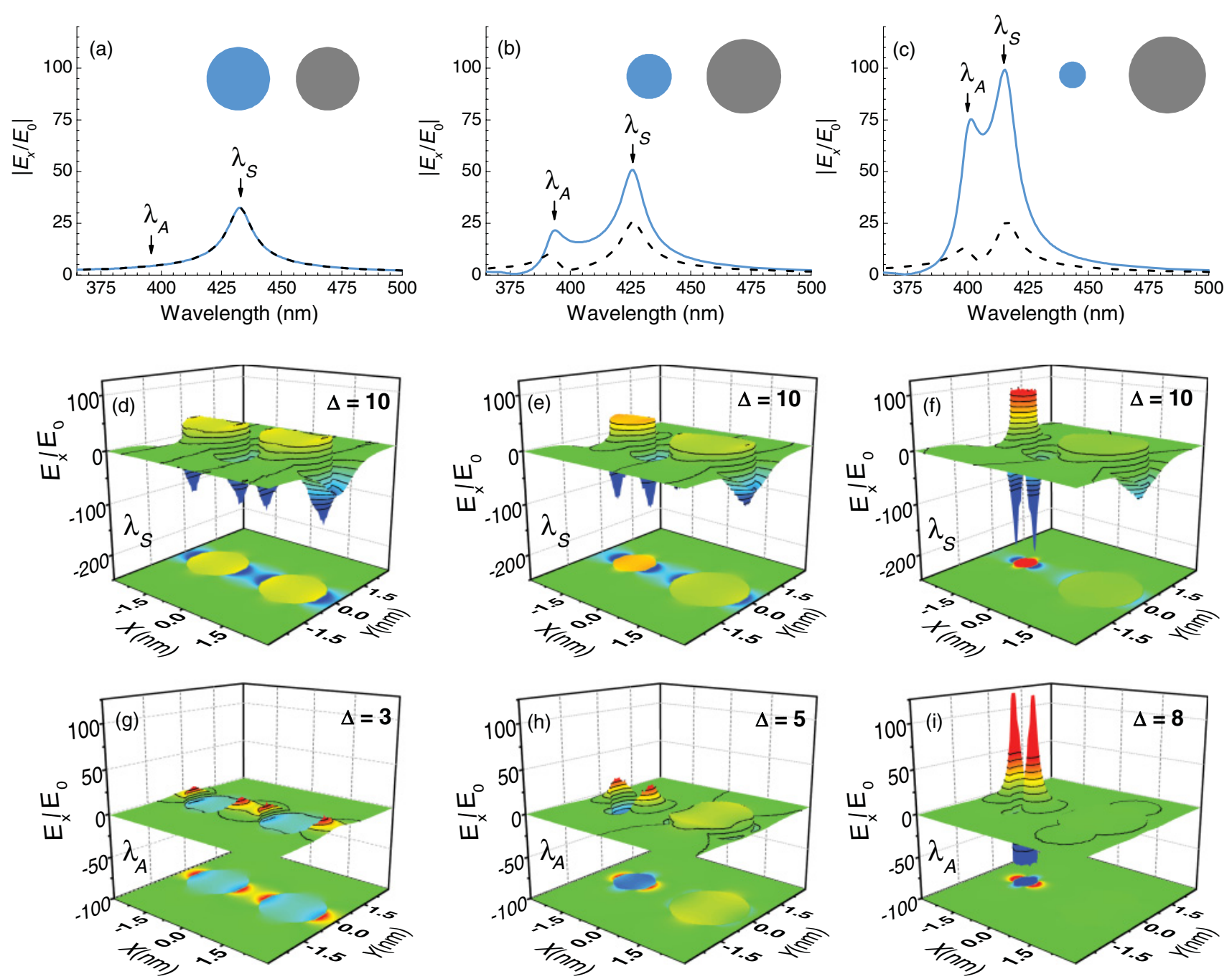

FIG. 3. (Color online) Magnitude of the electric field enhancement factor inside adjacent small (solid line) and large (dashed line) nanoparticles, for (a) identical sizes, (b) a volume ratio of 4.8, and (c) a volume ratio of 30, as well as the corresponding field enhancement distributions at a fixed phase for (d)-(f) the frequencies labeled $\lambda_{S}$ and for (g)-(i) the frequencies labeled $\lambda_{A}$. The field enhancement difference $\Delta$ between adjacent contour lines is indicated on each contour graph.

additional resonance increases in strength as the size difference grows, while its position gradually red-shifts.

To detect the presence of possible cascaded plasmon resonances, we monitor the frequency-dependent electric field enhancement factor $\left(E_{x} / E_{0}\right)$ at key points in the structure for three of the simulated geometries. Figures 3(a)-3(c) show the magnitude of the electric field enhancement factor at the center of the small nanoparticle (solid line) and of the large nanoparticle (dashed line) for arrays containing particles with a single particle size [Fig. 3(a)], as well as arrays containing dissimilar particles with a volume ratio of 4.8 [Fig. 3(b)] and 30 [Fig. 3(c)]. For the structure with a single particle size, the maximum field enhancement is identical in all particles and is a factor 33. Introducing a nearest-neighbor size difference leads to additional field enhancement in the smaller particle across a broad wavelength range. For the structure with the largest volume ratio [Fig. 3(c)], a maximum field enhancement of $97 \times$ is observed, approximately three times that observed in the structure with identically sized particles. This increased enhancement is indicative of a cascaded plasmon resonance, in which the smaller nanoparticle is excited predominantly by the enhanced near field around the larger nanoparticle, leading to additional field enhancement.

Figures 3(d)-3(f) show the distribution of the electric field enhancement $E_{x} / E_{0}$ at the excitation wavelengths marked $\lambda_{S}$ in Figs. 3(a)-3(c), respectively, shown at a fixed phase in the $x-y$ plane through the centers of the particles in the array. For identically sized particles [Fig. 3(d)], moderate field enhancement is observed both inside and outside the particles. At this frequency, the field inside adjacent particles has the same sign. This field distribution is referred to as the "symmetric mode." As the size difference increases [Figs. 3(e) and 3(f)], the field enhancement inside and around the smaller nanoparticles increases, while the field enhancement in the larger particle is relatively unaffected. These symmetric modes are responsible for the main absorption peak observed in Fig. 2. Interestingly the peak absorption coefficient for the symmetric mode (Fig. 2) only weakly depends on the volume ratio, likely 
because the increased internal field in the smaller particle is accompanied by a reduced volume over which the enhanced field is present.

Figures 3(g)-3(i) shows the spatial distribution of the field enhancement factor for these same structures for excitation at the wavelengths marked $\lambda_{A}$ in Figs. 3(a)-3(c), respectively. For identically sized particles [Fig. 3(g)], this wavelength is far from the plasmon resonance wavelength; consequently, only a small internal field enhancement is observed. As the size difference increases [Figs. 3(h) and 3(i)], the field enhancement in and around the smaller nanoparticles increases. In contrast to the results in Figs. 3(e) and 3(f), the field inside adjacent particles is opposite in sign. This mode is referred to as the "antisymmetric mode." For evenly spaced arrays with a single particle size, this mode cannot be excited by far-field illumination, in which case it represents a dark mode of the system. The introduction of alternating particle sizes introduces a periodicity in the array that matches that of the antisymmetric mode, allowing its far-field excitation. The appearance of this previously dark mode resembles effects observed in plasmonic electromagnetically induced transparency, which is due to the interaction of bright and dark modes in coupled metal resonators. ${ }^{35-37}$ Similar coupling effects that render dark modes weakly visible lead to the observation of Fano resonances in coupled plasmonic structures. ${ }^{38-42}$

The cascaded field enhancement observed in Fig. 3 suggests that these structures will exhibit a strongly enhanced nonlinear optical response at specific excitation wavelengths, at least for material located near the smaller particle. The overall enhancement of the nonlinear refractive and absorptive response of a Kerr-type composite can be extracted based on calculated linear field distributions and knowledge of the nonlinear susceptibilities of the materials in the structure. Under excitation of the structure with $x$-polarized light at a single frequency, the effective third-order nonlinear susceptibility of the composite $\chi_{\text {eff }}{ }^{(3)}$ at the fundamental can be written as

$$
\chi_{\mathrm{eff}}^{(3)}(\omega)=\sum f_{j} g_{j}^{(3)}(\omega) \chi_{j}^{(3)}(\omega)
$$

where $f_{j}$ and $\chi_{j}{ }^{(3)}(\omega)$ are the fill fraction and third-order susceptibility of material $j$ (here labeled "in" for the particle and "out" for the host material) and $g_{j}{ }^{(3)}(\omega)$ is a complex, thirdorder nonlinear susceptibility enhancement factor derived from the simulated frequency-dependent linear electric field distribution $\vec{E}(\omega, r)$ and is given by ${ }^{18,43}$

$$
g_{j}^{(3)}=\frac{\left\langle\vec{E}^{2}|\vec{E}|^{2}\right\rangle_{V_{j}}}{\langle\vec{E}\rangle_{V}^{2}\left|\langle\vec{E}\rangle_{V}\right|^{2}}
$$

where the notations $\langle\ldots\rangle_{V_{j}}$ and $\langle\ldots\rangle_{V}$ indicate spatial averaging over the volume of component $j$ and over the total simulation volume, respectively. Frequency and position arguments have been omitted for simplicity of notation. The complex nature of the enhancement factors manifests itself predominantly around resonances, where local fields occur with significant phase delays relative to the incident field.

Figure 4 shows the phase and magnitude of the obtained third-order nonlinear susceptibility enhancement factor $g_{\text {in }}{ }^{(3)}$ of the nonlinear response of the nanoparticles. For identically sized particles (curve E), a maximum enhancement of $2.0 \times 10^{6}$ is obtained at the wavelength corresponding to

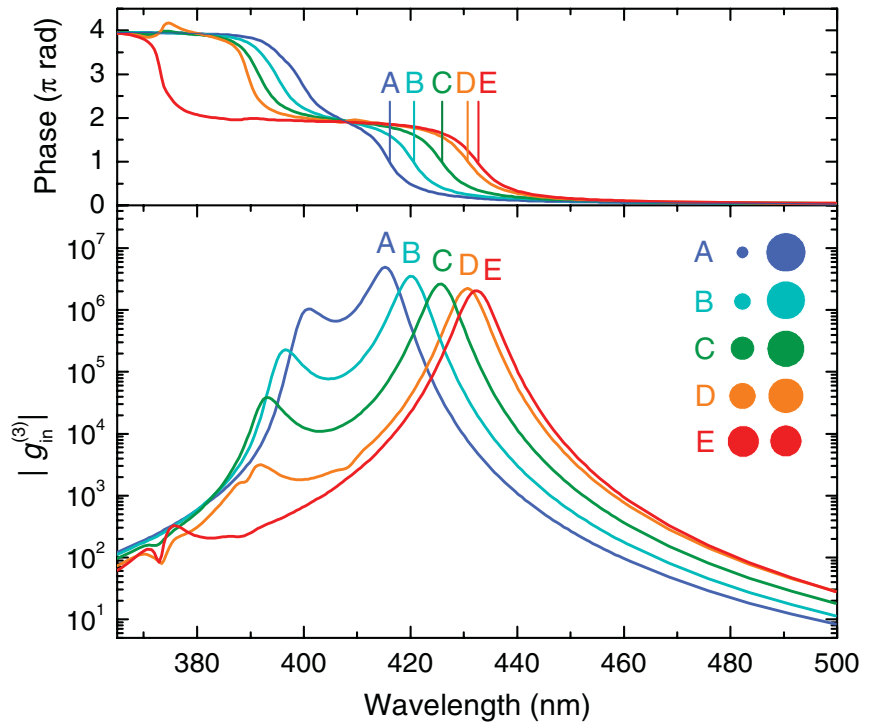

FIG. 4. (Color online) (a) Phase and (b) magnitude of the internal, complex nonlinear susceptibility enhancement factor for nearestneighbor volume ratios of $1,2.3,4.8,11$, and 30 . The legend shows the corresponding unit cell of the periodic metamaterial.

the symmetric resonance $(\lambda=432 \mathrm{~nm})$. The phase of the enhancement at this frequency is close to $\pi$. This implies that plasmon resonant particles composed of a metal with large positive imaginary $\chi_{\text {in }}{ }^{(3)}$ produce a composite response $\chi_{\text {eff }}{ }^{(3)}=$ $f_{\text {in }} g_{\text {in }}{ }^{(3)} \chi_{\text {in }}{ }^{(3)}$ that is large and negative, resulting in the appearance of saturable absorption at the plasmon resonance wavelength.

As the size difference between nearest neighbors increases, the magnitude of the enhancement for the symmetric mode gradually increases by a factor 2.4 , while the phase at the wavelength for maximum enhancement remains approximately $\pi$. In addition, the introduction of a finite size difference leads to a nonlinear susceptibility enhancement at wavelengths near the antisymmetric plasmon resonance. This enhancement is much weaker than that of the symmetric mode; however, its magnitude increases rapidly as the size difference is increased. The phase of the enhancement factor reaches values close to $3 \pi$ at the antisymmetric resonance frequency, physically equivalent to the phase response at the symmetric mode wavelength. However, at wavelengths between the symmetric and the antisymmetric resonances, the enhancement factor remains high while exhibiting a phase close to $\sim 2 \pi$. This represents a new enhancement regime that does not occur in the composite with only a single particle size. At these wavelengths, plasmon resonant metals with a predominantly imaginary $\chi^{(3)}$ response could be used to obtain composites with a positive imaginary $\chi_{\text {eff }}{ }^{(3)}$, corresponding to enhanced nonlinear absorption. This frequency range also corresponds to a region of low linear absorption (Fig. 2), suggesting that simultaneous enhancement of nonlinear absorption and improvement of the useful interaction length can be achieved in a single composite design.

To understand the observations in Figure 4, we consider nonlinear optical absorption as the consequence of a perturbed linear response. At high irradiance, the absorption spectrum of the composite is expected to undergo small changes due to 


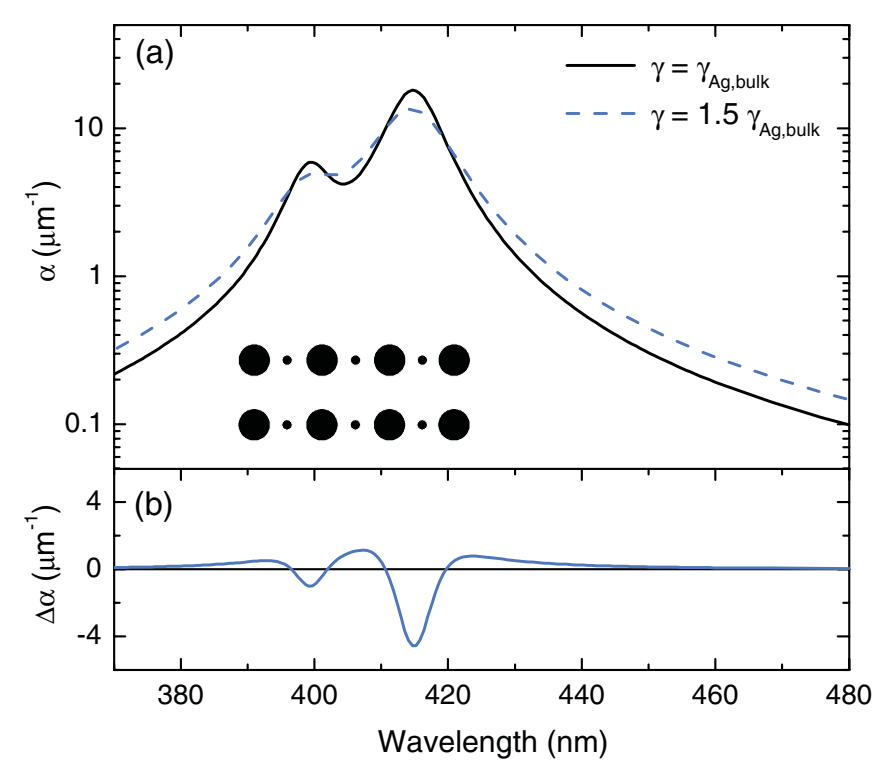

FIG. 5. (Color online) (a) Absorption coefficient of a cascaded plasmonic metamaterial with a volume ratio of 30 calculated using a Drude fit to the literature data for the silver dielectric function (solid line) and of the same structure with the electron scattering rate $\gamma_{\mathrm{Ag}}$ artificially increased by $50 \%$ (dashed line). (b) Difference between the curves in (a), indicating reduced absorption at the resonance peaks and increased absorption at frequencies between the resonances.

nonlinearly induced modification of the polarizability of the materials involved. The addition of a small imaginary part to the metal dielectric function, which is expected to occur at high irradiance, leads to broadening of plasmon resonances in the system. To illustrate this effect, Fig. 5(a) shows the calculated absorption spectrum of a structure with volume ratio 30 (solid line), and the absorption spectrum of the same structure where the electron scattering rate $\gamma_{\mathrm{Ag}}$ has artificially been increased by $50 \%$ (dashed line), to mimic the metal nonlinear response. The resulting change in the dielectric function leads to broadening of the symmetric and antisymmetric modes, as well as a change in the peak absorption values. This effect causes a rise in the absorption coefficient in the area between the two resonance peaks, corresponding to the previously predicted composite nonlinear absorption.

The performance of nonlinear optical refractive or absorptive materials is typically evaluated in terms of a FOM, often defined as $\left|\chi^{(3)}\right| / \alpha$, or in the specific case of nonlinear absorption as $\beta / \alpha$, where $\beta$ is the nonlinear absorption coefficient. Due to the complex nature of both $\chi^{(3)}$ and thirdorder susceptibility enhancement factors $g^{(3)}$, it is nontrivial to assign a generally applicable FOM. Here, we define a complex enhancement FOM given by $g^{(3)} / \alpha$, which represents the ability of a given cascaded plasmon resonant metamaterial to enhance the third-order nonlinear optical response. Figure 6 shows the obtained complex enhancement FOM for the array with a single particle size and for the array with a nearestneighbor volume ratio of 30 , displayed on the same relative scale. The symbols correspond to simulated data points, while the solid lines provide a guide to the eye. For the structure containing particles with a single size (smaller inner red curve), the largest enhancement FOM occurs at a complex phase of $\pi$

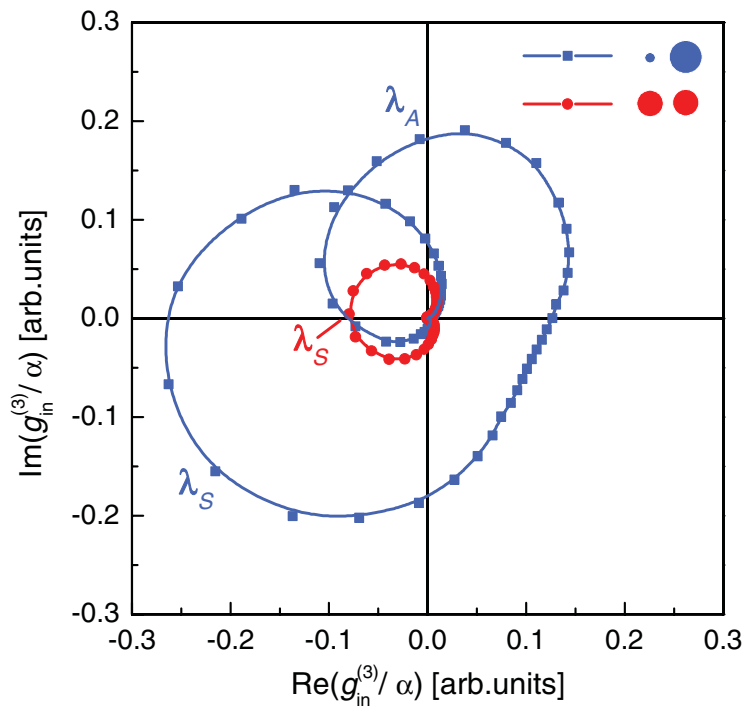

FIG. 6. (Color online) Complex enhancement FOM of the third-order nonlinear optical response of a plasmonic metamaterial containing silver nanoparticles with a single size (radius $=1 \mathrm{~nm}$ ) and of a cascaded plasmonic metamaterial with a nearest-neighbor volume ratio of 30 . The legend shows the corresponding unit cell of the periodic metamaterial. The labels $\lambda_{A}$ and $\lambda_{S}$ correspond to the antisymmetric and symmetric modes, respectively.

corresponding to a predominantly real negative enhancement factor. This occurs at the main dipolar plasmon resonance wavelength of the structure, marked $\lambda_{S}$ in Fig. 3(a). The cascaded structure with a volume ratio of 30 (larger outer blue curve), however, shows a different range of complex enhancement FOM values. First, at any complex phase, the cascaded structure can provide an improved enhancement FOM. Second, a real and positive enhancement FOM is obtained in the cascaded structure, occurring at frequencies between the symmetric and the antisymmetric modes. This shows that despite the relatively low field enhancement at wavelengths between $\lambda_{A}$ and $\lambda_{S}$, the corresponding moderate linear absorption at these intermediate wavelengths enables a FOM that dramatically exceeds that of the noncascaded metamaterial.

To illustrate the effect of the complex enhancement factors that can be achieved in cascaded plasmon resonant structures, Fig. 7 shows a specific example evaluating the FOM for nonlinear absorption, given by $\beta / \alpha$, with $\beta$ as the nonlinear absorption coefficient. We assume that the metal $\chi^{(3)}$ response is well described by a Kerr-type response with a predominantly positive imaginary response given by $\operatorname{Im}\left(\chi^{(3)}\right)=10^{-16} \mathrm{~m}^{2} / \mathrm{V}^{2}$. For the array with a single particle size, this leads to a small positive FOM for nonlinear absorption at frequencies above and below the plasmon resonance, while a large and negative FOM is found at the plasmon resonance frequency, indicative of saturable absorption rather than enhanced nonlinear absorption. This behavior can be understood in terms of a nonlinearly induced broadening of the plasmon resonance, using a similar analysis to that shown in Fig. 5. As the particle size difference within the array increases, both the antisymmetric and the symmetric modes produce an increasingly strong negative FOM. However, at wavelengths between the two resonances, a 


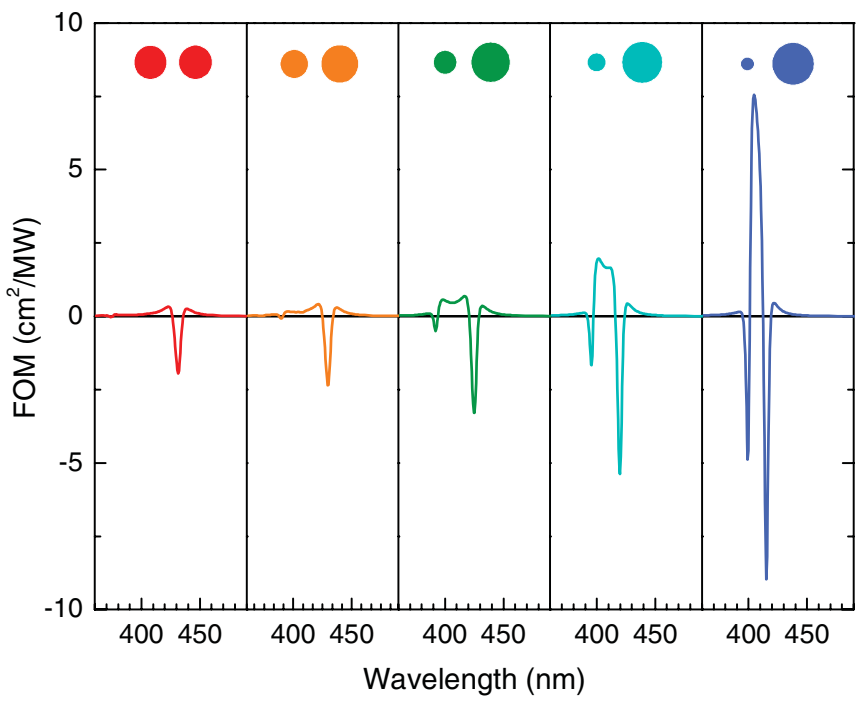

FIG. 7. (Color online) Calculated FOM for nonlinear absorption assuming $\operatorname{Im}\left(\chi^{(3)}\right)=10^{-16} \mathrm{~m}^{2} / \mathrm{V}^{2}$ for cascaded plasmon resonant metamaterials with different nearest-neighbor particle volume ratios of $1,2.3,4.8,11$, and 30 . The corresponding unit cell of the period structures is indicated schematically. An improvement of the FOM for nonlinear absorption by a factor 20 is observed as the nearest-neighbor volume ratio is increased from 1 to 30 .

rapidly increasing positive FOM develops, ultimately reaching a value that is larger by a factor of 20 than the maximum value observed using a single particle size. Depending on the excitation pulse length and frequency, $\chi_{\text {in }}{ }^{(3)}$ can take on various complex phases and magnitudes; thus, Fig. 7 should be taken primarily as a demonstration of the practical implications of the complex nature of the enhancement factors. Nevertheless, effects such as those in Fig. 7 are predicted to be observable in frequency-dependent nonlinear optical measurements on cascaded plasmon resonant structures.

The results shown previously were obtained in the quasielectrostatic limit in the absence of surface scattering. At the small particle sizes simulated here, surface scattering would significantly alter the surface plasmon damping, leading to reduced field enhancement factors and lower FOMs for nonlinear refraction and absorption, albeit over a larger frequency bandwidth. For this reason, any experimental implementation of the discussed approach would require larger particle sizes and likely larger interparticle spacing. Similar calculations in which all dimensions are scaled up by a factor 10 (not shown) yield similar results in both linear and nonlinear optical response while remaining in the effective medium limit (no diffractive effects are present in the frequency region of interest). These results strongly suggest that the predicted response can be obtained using experimentally viable particle sizes with reduced surface scattering effects.

Finally, while these effects were calculated assuming pure Kerr-type nonlinearities, the fundamental physical interactions that produce the effects discussed here are related to the presence of coupled nonlinear resonators. Consequently, similar enhancement effects are expected to appear in systems that exhibit non-Kerr-type, third-order optical nonlinearities, including thermal nonlinearities.

\section{CONCLUSIONS}

The use of cascaded plasmon resonances for the enhancement of nonlinear optical refraction and absorption was found to lead to complex enhancement factors and complex enhancement FOMs that strongly depend on the nearest-neighbor volume ratio. Cascaded plasmon resonances were found to increase the attainable nonlinear susceptibility enhancement factors and FOMs for nonlinear absorption. The plasmon cascading effect was demonstrated to offer the possibility of obtaining large nonlinear optical absorption using metals with a positive imaginary third-order nonlinear optical susceptibility.

\section{ACKNOWLEDGMENTS}

This material is based upon work supported in part by the US Army Research Office under Contract/Grant No. 50372CH-MUR. *kik@creol.ucf.edu

${ }^{1}$ K. Li, M. I. Stockman, and D. J. Bergman, Phys. Rev. B 72, 153401 (2005).

${ }^{2}$ S. Kujala, B. K. Canfield, M. Kauranen, Y. Svirko, and J. Turunen, Phys. Rev. Lett. 98, 167403 (2007).

${ }^{3}$ B. K. Canfield, H. Husu, J. Laukkanen, B. F. Bai, M. Kuittinen, J. Turunen, and M. Kauranen, Nano Lett. 7, 1251 (2007).

${ }^{4}$ M. Lippitz, M. A. van Dijk, and M. Orrit, Nano Lett. 5, 799 (2005).

${ }^{5}$ T. Utikal, T. Zentgraf, T. Paul, C. Rockstuhl, F. Lederer, M. Lippitz, and H. Giessen, Phys. Rev. Lett. 106, 133901 (2011).

${ }^{6}$ J. E. Sipe and R. W. Boyd, Phys. Rev. A 46, 1614 (1992).

${ }^{7}$ N. N. Lepeshkin, W. Kim, V. P. Safonov, J. G. Zhu, R. L. Armstrong, C. W. White, R. A. Zuhr, and V. M. Shalaev, J. Nonlinear Opt. Phys. Mater. 8, 191 (1999).

${ }^{8}$ D. C. Kohlgraf-Owens and P. G. Kik, Opt. Express 16, 16823 (2008).
${ }^{9}$ G. Piredda, D. D. Smith, B. Wendling, and R. W. Boyd, J. Opt. Soc. Am. B 25, 945 (2008).

${ }^{10}$ K. Uchida, S. Kaneko, S. Omi, C. Hata, H. Tanji, Y. Asahara, A. J. Ikushima, T. Tokizaki, and A. Nakamura, J. Opt. Soc. Am. B 11, 1236 (1994).

${ }^{11}$ K. H. Kim, A. Husakou, and J. Herrmann, Opt. Express 18, 7488 (2010).

${ }^{12}$ H. B. Liao, W. Wen, and G. K. L. Wong, Appl. Phys. Mater. Sci. Process. 80, 861 (2005)

${ }^{13}$ N. Pinçon, B. Palpant, D. Prot, E. Charron, and S. Debrus, Eur. Phys. J. Atom. Mol. Opt. Phys. 19, 395 (2002).

${ }^{14}$ E. L. Falcao-Filho, C. B. de Araujo, A. Galembeck, M. M. Oliveira, and A. J. G. Zarbin, J. Opt. Soc. Am. B 22, 2444 (2005).

${ }^{15}$ O. Maruyama, Y. Senda, and S. Omi, J. Non-Cryst. Solids 259, 100 (1999). 
${ }^{16}$ G. Ma, W. Sun, S.-H. Tang, H. Zhang, Z. Shen, and S. Qian, Opt. Lett. 27, 1043 (2002).

${ }^{17}$ J. Jayabalan, A. Singh, R. Chari, and S. M. Oak, Nanotechnology 18, 315704 (2007).

${ }^{18}$ D. C. Kohlgraf-Owens and P. G. Kik, Opt. Express 17, 15032 (2009).

${ }^{19}$ A. I. Fernandez-Dominguez, S. A. Maier, and J. B. Pendry, Phys. Rev. Lett. 105, 266807 (2010).

${ }^{20}$ D. J. Bergman, Phys. Rev. B 19, 2359 (1979).

${ }^{21}$ D. J. Bergman, J. Phys. Solid State Phys. 12, 4947 (1979).

${ }^{22}$ X. Zhang, K. H. Su, Q. H. Wei, J. J. Mock, D. R. Smith, and S. Schultz, Nano Lett. 3, 1087 (2003).

${ }^{23}$ A. Leitner, W. Rechberger, A. Hohenau, J. R. Krenn, B. Lamprecht, and F. R. Aussenegg, Opt. Commun. 220, 137 (2003).

${ }^{24}$ P. Nordlander, C. Oubre, E. Prodan, K. Li, and M. I. Stockman, Nano Lett. 4, 899 (2004).

${ }^{25}$ A. Wei, D. A. Genov, A. K. Sarychev, and V. M. Shalaev, Nano Lett. 4, 153 (2004).

${ }^{26}$ A. V. Nurmikko, T. Atay, and J. H. Song, Nano Lett. 4, 1627 (2004).

${ }^{27}$ F. J. Garcia de Abajo, S. Riikonen, and I. Romero, Phys. Rev. B 71, 235104 (2005).

${ }^{28}$ U. Hohenester and J. R. Krenn, Phys. Rev. B 72, 195429 (2005).

${ }^{29}$ A. Aubry, D. Y. Lei, S. A. Maier, and J. B. Pendry, Phys. Rev. Lett. 105, 233901 (2010).

${ }^{30}$ K. Li, M. I. Stockman, and D. J. Bergman, Phys. Rev. Lett. 91, 227402 (2003).
${ }^{31}$ V. G. Kravets, G. Zoriniants, C. P. Burrows, F. Schedin, C. Casiraghi, P. Klar, A. K. Geim, W. L. Barnes, and A. N. Grigorenko, Phys. Rev. Lett. 105, 246806 (2010).

${ }^{32}$ K. Li, M. I. Stockman, and D. J. Bergman, Phys. Rev. Lett. 97, 079702 (2006).

${ }^{33}$ Microwave Studio 2011, Computer Simulation Technology, Darmstadt, Germany.

${ }^{34}$ S. A. Maier, M. L. Brongersma, P. G. Kik, and H. A. Atwater, Phys. Rev. B 65, 193408 (2002).

${ }^{35}$ V. Yannopapas, E. Paspalakis, and N. V. Vitanov, Phys. Rev. B 80, 035104 (2009).

${ }^{36}$ N. Liu, L. Langguth, T. Weiss, J. Kastel, M. Fleischhauer, T. Pfau, and H. Giessen, Nat. Mater. 8, 758 (2009).

${ }^{37}$ S. Zhang, D. A. Genov, Y. Wang, M. Liu, and X. Zhang, Phys. Rev. Lett. 101, 047401 (2008).

${ }^{38}$ F. Hao, P. Nordlander, Y. Sonnefraud, P. Van Dorpe, and S. A. Maier, ACS Nano 3, 643 (2009).

${ }^{39}$ N. Verellen, Y. Sonnefraud, H. Sobhani, F. Hao, V. V. Moshchalkov, P. Van Dorpe, P. Nordlander, and S. A. Maier, Nano Lett. 9, 1663 (2009).

${ }^{40}$ J. B. Lassiter, H. Sobhani, J. A. Fan, J. Kundu, F. Capasso, P. Nordlander, and N. J. Halas, Nano Lett. 10, 3184 (2010).

${ }^{41}$ B. Luk'yanchuk, N. I. Zheludev, S. A. Maier, N. J. Halas, P. Nordlander, H. Giessen, and C. T. Chong, Nat. Mater. 9, 707 (2010).

${ }^{42}$ Y. Sonnefraud, N. Verellen, H. Sobhani, G. A. E. Vandenbosch, V. V. Moshchalkov, P. Van Dorpe, P. Nordlander, and S. A. Maier, ACS Nano 4, 1664 (2010).

${ }^{43}$ D. Stroud and P. M. Hui, Phys. Rev. B 37, 8719 (1988). 